J. Biosoc. Sci., (2016) 48, S74-S91 (C) Cambridge University Press, 2016. This is an Open Access article, distributed under the terms of the Creative Commons Attribution licence (http:// creativecommons.org/licenses/by/4.0/), which permits unrestricted re-use, distribution, and reproduction in any medium, provided the original work is properly cited.

doi:10.1017/S0021932016000237

\title{
LOW PREVALENCE OF INTESTINAL SCHISTOSOMIASIS AMONG FISHERFOLK LIVING ALONG THE RIVER NILE IN NORTH-WESTERN UGANDA: A BIOSOCIAL INVESTIGATION
}

\author{
GEORGINA PEARSON ${ }^{1}$ \\ Department of International Development, London School of Economics and \\ Political Science, UK
}

\begin{abstract}
Summary. Mass drug administration has been less successful as a technique for controlling intestinal schistosomiasis ( $S$. mansoni) than anticipated. In Uganda, the mass distribution of praziquantel has been provided to populations at risk of infection since the early 2000s, but prevalence mostly remains high. This is the case, for example, at locations in north-western and south-eastern Uganda. However, there is a remarkable exception. Among Madi fishing populations and their immediate neighbours, living close to the border with South Sudan, the rate of infection has dropped dramatically. A parasitological survey carried out at twelve fishing sites in 2013 identified only three cases of $S$. mansoni among 383 adults tested. This article asks: why is the prevalence of $S$. mansoni so low among fisherfolk in northern Uganda? Taking a biosocial approach, it suggests that the mass distribution of drugs, free of charge, has had an impact. However, the low prevalence of infection cannot be attributed to this alone. Other important factors may also have contributed to the decline in infection. These include changing fishing livelihoods, local attitudes to public health interventions, access to water and sanitation facilities, hygiene practices and the use of anti-malarial treatments. Above all, the article highlights the importance of investigating both social and biological dimensions of infection simultaneously, and of recognizing the local complexities of sustainably treating this debilitating parasitic disease.
\end{abstract}

\section{Introduction}

Schistosomiasis is a neglected tropical disease, associated with poverty. It predominantly affects rural populations living in tropical and sub-tropical environments. The World Health Organization $(2012,2016)$ estimates that over 200 million people worldwide are

${ }^{1}$ Email: g.f.pearson@1se.ac.uk 
infected with schistosomiasis, with over $90 \%$ of those infected or at risk of infection living in the African continent. A number of species of schistosome cause disease in humans (S. mansoni, S. haematobium, S. japonicum, S. mekongi and S. intercalatum). This article focuses on $S$. mansoni, the species that is endemic in northern and northwestern Uganda. Schistosoma mansoni can have varying health effects, ranging from acute intestinal symptoms to chronic complications affecting the liver and spleen. Biologically, the transmission of $S$. mansoni occurs when faeces containing the parasite eggs contaminate rivers and lakes. The eggs produce miracidia that enter and develop inside suitable fresh water snail hosts. They then release microscopic cercariae into the water which enter the human body through the skin when a person comes in contact with infested water (see Gryseels et al., 2006). Transmission is, therefore, influenced by broader social determinants beyond the biological life cycle, and disease patterns show microgeographical variations, with interactions between biological, social, behavioural and environmental factors determining vulnerability to schistosomiasis (Grimes et al., 2015).

Following recommendations by the World Health Organization to treat $75 \%$ of school-aged children in highly endemic areas (WHO, 2002), annual mass drug administration with praziquantel was introduced in Uganda from 2003 (Kabatereine et al., 2006). The primary aim was to interrupt or reduce transmission and, thereby, reduce morbidity from schistosomiasis by distributing praziquantel to all school-aged children and adults living in endemic areas, irrespective of their infection status. Implementing mass drug administration has been challenging. The majority of research in Uganda has been carried out along Lake Victoria and Lake Albert where infection rates have remained high. Local-level research has found that among people along the lakeshores there is often low drug coverage and, in some cases, there has been resistance to the distribution (Parker et al., 2008; Allen \& Parker, 2011; Parker \& Allen, 2011, 2014; Muhumuza et al., 2013, 2015). Despite multiple rounds of free distribution of praziquantel, high rates of reinfection in both adults and children have persisted, even up to $80-90 \%$ at some locations (Kabatereine et al., 2011; Seto et al., 2012; Allen \& Parker, 2016). In this context, it is remarkable to find that the prevalence of $S$. mansoni is so low among fisherfolk living in areas of north-western Uganda along the River Nile to the north of Rhino Camp and the border with South Sudan. Here, transmission and prevalence appear to have significantly declined.

Although historical data on rates of infection in the vicinity prior to mass drug administration are inconsistent and confusing, they all suggest that they have been relatively high. Nelson (1958, cited in Bradley, 1975, p. 51) conducted S. mansoni surveys between 1951 and 1954 and estimated an average infection rate in West and East Madi (now Moyo and Adjumani Districts respectively) of more than 50\%. A rate of nearly 30\% was reported from one stretch of the river (Nelson, reported in WHO, 1987). Along another part of the river, a prevalence of over $80 \%$ was found in the early 1990 s, but this declined to 30\% following treatment with praziquantel (Frenzel et al., 1999; Odongo-Aginya et al., 2002). Similar figures were again reported for the same area of the Nile between 2005 and 2006 (Odongo-Aginya et al., 2010). A survey of Madi adults carried out under the auspices of the Vector Control Division of the Ugandan Ministry of Health in Laropi, Moyo District, reported a rate of just over $40 \%$ in 2003 . Then, one year later, after the first round of mass treatment, adult prevalence was reported to have fallen to $5.6 \%$ (Parker et al., 2012). 
The Ministry of Health's Vector Control Division has subsequently run a series of further surveys on school children, predominantly in Moyo District. These unpublished data (which go up to 2011) suggest that the overall prevalence of S. mansoni has continued to decline and, with few exceptions, remains low. Six primary schools in Moyo District were surveyed annually between 2003 and 2007. The overall prevalence was $20.1 \%$ in 2003 , with cases found at every school surveyed (and the prevalence ranging from $7.6 \%$ to $35 \%$ ). In 2007 , the overall prevalence fell to $9.6 \%$, and while it remained high at some schools $(32.7 \%)$, at other schools, no cases of infection with S. mansoni were found. Fewer surveys have included adults. However, in 2008, two subcounties in Moyo District along the River Nile were surveyed. Five communities were sampled in one sub-county, and the overall prevalence was 3.6\% (ranging from 0 to $12.5 \%$ ). In the other sub-county, four communities were sampled, and the overall prevalence was $29.2 \%$ (ranging from 0 to $66.7 \%$ ). The most recent survey carried out in the two districts was in 2011. In Adjumani, the overall prevalence was 9.6\% in the nine primary schools surveyed, and ranged from 0 to $26 \%$. In Moyo, in the nine primary schools surveyed, the overall prevalence was $2.5 \%$ and ranged from $0 \%$ to $8 \%$, demonstrating a further reduction from the 2003 to 2007 surveys by the Ministry of Health's Vector Control Division. Overall, these data suggest that over the course of mass drug administration, the overall prevalence of schistosomiasis has decreased and while $S$. mansoni was initially being found at all sites surveyed, at some sites in later surveys, no cases were identified.

It is worth noting that a small study carried out in one Madi fishing island in the Nile in 2009 confirmed the trend. Only one case of S. mansoni was found out of 31 adults tested (Parker et al., 2012). The research was based on limited parasitological data, but the findings were still surprising given that on the island there were no sanitation facilities or safe water provision and it was a busy fishing ground with people having daily contact with river water. Additionally, those adults tested spent the majority of their time in fishing-related activities, moving between landing sites.

To see if this low infection rate was more widespread, further research (which is presented in this article) was conducted between 2013 and 2014. Parasitological research documenting the prevalence of $S$. mansoni among adults living and working along the river took place at twelve landing sites. It was carried out while undertaking long-term ethnographic fieldwork exploring local understandings of several neglected tropical diseases. As described above, previous studies on schistosomiasis in this area have largely focused either on the parasitological aspects of infection or the social responses to mass drug administration. The research presented in this article expands on these studies by analysing the parasitological findings in the light of long-term ethnographic fieldwork exploring the local context of schistosomiasis transmission and control. In so doing, it is shown that while preventive chemotherapy is likely to have had some impact on disease rates, the low prevalence cannot be attributed to mass drug administration alone. It needs to be understood within the context of other biological and social factors that influence vulnerability to schistosomiasis and affect control. Use of anti-malarials that have additional anti-schistosome effects, aspects of movement and livelihoods in this border area, and water, sanitation and hygiene practices at the landing sites are, therefore, examined. While findings on $S$. mansoni prevalence among the Madi and nearby fishing populations are encouraging, sustaining control over time will require 
a reconsideration of the social and structural drivers affecting risk. Also, recognizing that there are multiple reasons for the low prevalence highlights the need for similar biosocial approaches to assessment in other parts of Uganda where prevalence remains high.

\section{Moyo and Adjumani Districts, north-western Uganda}

Moyo and Adjumani Districts in north-western Uganda are separated by the River Nile and border South Sudan. Geographically, the districts differ. Moyo is a mountainous region with a lowland area along the river, whereas Adjumani is predominantly savannah grassland. Many neglected tropical diseases are endemic in the districts, including S. mansoni, soil-transmitted helminths, lymphatic filariasis, onchocerciasis and human African trypanosomiasis (sleeping sickness). District vector control officers oversee the mass distribution of drugs (which occurs for the first four of the diseases named above), as well as vector surveillance activities for onchocerciasis and sleeping sickness. There have been numerous public health programmes for some of these diseases in the past, with sleeping sickness control being particularly prominent (see, for example, Allen \& Storm, 2012).

The region has a long history of social upheaval as well as political and economic marginalization (Allen, 1991; Woodward, 1991; Allen \& Storm, 2012), dating back to slave and ivory traders in the nineteenth century, through the colonial period to independence. Following independence in 1962, there were decades of conflict and during the 1980s the majority of the population in both districts fled into southern Sudan (now South Sudan) or Zaire (now Democratic Republic of Congo, DRC), returning in 1986. In the 1990s and 2000s, a large number of refugees from southern Sudan settled in both districts but the majority repatriated by 2009. However, the situation changed again in December 2013 when tens of thousands of refugees from South Sudan fled into Adjumani District.

A preliminary report from the most recent census in 2014 estimated the population of Adjumani to be 232,813 and that of Moyo to be 137,489 (UBOS, 2014, pp. 20 and 21). The majority of people in Moyo and Adjumani Districts are from Madi clans and speak the language of the same name, Madi. However, in Obongi County (which is situated along the river in Moyo District), people are from many ethnic groups and identify themselves as Aliba, Aringa, Gimara and Reli. In these sub-counties, many languages are spoken in addition to Madi. In all the sub-counties, some people speak English (the official language in government and the language used in education from primary school grade four onwards) and Kiswahili.

The majority of people are subsistence farmers, growing crops such as cassava, sweet potato, maize, groundnuts, sesame and various green-leafed vegetables. Rice, pumpkins and tomatoes are grown in swamp areas along the river banks. Fish is relatively expensive in the town, but part of the daily diet at the river. There are also large areas for cattle to graze. People are drawn to livelihoods along the river with its fertile land, food sources and economic opportunities.

There are two main river crossing points for vehicles - Laropi and Sinyanya - both of which are served by a Uganda National Roads Authority ferry. At many other sites, people, plus their luggage, livestock, bicycles and motorbikes cross by motorboat or 
rowed canoe. Motorboats also cross from both districts to Nimule, a busy border market town in South Sudan where a number of fishermen and fishmongers sell their fish. This was a popular trading route, and during fieldwork in 2013 and 2014 an increasing number of motorboats served these crossings.

Fish is important economically with a dynamic local market. Fishing itself is, with very few exceptions, a male occupation. Women are largely responsible for the smoking and curing of fish. Female fishmongers either sell fresh fish directly at nearby markets, or more often, dry the fish over a smoking oven first. They take the dried fish to markets either in the main towns in the districts or to Nimule. Male fishmongers who own motorbikes tend to buy fresh fish and sell it directly at markets that same day. They sell it in Adjumani or Moyo towns, or further afield in West Nile in places such as Arua, Yumbe and Koboko. Sometimes they employ women at the landing sites to clean the fish, paying each woman 1000 Ugandan shillings (around US\$0.40) per day for their labour.

Ethnographic fieldwork focused on people living and working at the fish landing sites (oti). Rather than the urbanizing sites along Lake Victoria that have been described by Beuving (2010), in Moyo and Adjumani the fish business operates on a smaller scale, with the majority of sites being village landing sites or fishing camps. Fishermen and female fishmongers stay at the landing sites for days, weeks or months at a time, in small grass-thatched houses (tukuls), under temporary shelters or out in the open. One of the sites visited during fieldwork could only be reached by land in the dry months (January to March); the rest of the year it was an island that was reached by boat. There are numerous islands and reed banks in the river where fishermen stay overnight, sometimes for weeks at a time, before returning to the mainland. In addition to the fish business, the fish landing sites are used for many domestic purposes such as collecting water and washing clothes. At some sites, people operate small businesses, selling cooked food, tea, soda, alcohol, soap and cigarettes.

\section{Methods}

Building on three months of fieldwork in Moyo District in 2009 (Pearson, 2009), further epidemiological and ethnographic fieldwork in Moyo and Adjumani Districts was undertaken over twelve months in 2013 to 2014. The ethnographic fieldwork included participant-observation, group discussions, semi-structured interviews and open-ended unstructured interviews with key informants at the landing sites (such as fishermen, fish-processors, fishmongers, local council members, Beach Management Unit members and elders) as well as health workers, local healers and people working for district authorities (particularly in health and fisheries). These interviews and discussions were not recorded, but notes were taken at the time and written up in full afterwards. Field notes based on participant-observation were also written up at the end of each day.

From the end of October to December 2013, a cross-sectional survey (in collaboration with staff from district vector control) was conducted at twelve fishing sites with a team of two research assistants and four laboratory technicians. With limited baseline data on local disease rates (especially at the fishing sites), a broad geographical area was surveyed and fishing sites along an $80 \mathrm{~km}$ stretch of the river were purposefully chosen to represent the sub-counties along the Nile. There were a few 
exceptions, but at the majority of these sites, it is predominantly adults who frequent the sites and are involved in the fish business, and at some fishing sites, according to local by-laws, children are not allowed. Thus, the survey focused on adults.

Three hundred and eighty-three adults participated in the survey (203 males and 180 females). They were aged from 18 to 75 years, with an average age of 36 years. Around 30-50 adults participated from each site with roughly equal numbers of men and women. As the people at the landing sites were constantly changing, baseline population data for the landing sites were difficult to collect. Furthermore, convenience sampling was adopted as random sampling was not feasible. As one member of the team observed, commenting on attempts to organize random sampling: 'It is difficult to make these fishers wait; you must take them when you can.' The objective was to enable adults who would ordinarily miss such programmes in their home village (because of their involvement in the fish business) to participate.

Experienced research assistants interviewed the participants in the local language using a structured questionnaire. This questionnaire documented demographic details of each participant, including their age, sex, education, ethnic group, occupation and how many children they had. In addition, details were obtained on medication use (praziquantel for schistosomiasis and the artemisinin derivative Coartem for malaria), use of local herbs, health status, water and latrine use, and factors relating to the fish business (the landing sites used, time of day at landing sites, fishing methods used, frequency of using the landing sites and the number of years spent fishing or in related occupations).

Information about the study was explained to the participants in their language, and they provided a signature or a thumbprint (if they could not read or write) to confirm their consent. Each participant provided a stool sample, and the technicians prepared and examined two Kato-Katz slides (with malachite green) from each sample. The Kato-Katz method is the current recommendation for mapping intestinal schistosomiasis (Lamberton et al., 2014), and the approach used in many studies. However, it is important to note that in low-transmission areas, low-intensity infections may be missed (King, 2010), with Lamberton et al. (2014) reporting a sensitivity of $74 \%$ for two Kato-Katz smears at six months post-praziquantel. At the time of the survey, other diagnostic techniques (for example, urine antigen testing, discussed in Lamberton et al. (2014)) were not available; and other methods (for example analysing six Kato-Katz slides per person, that is, two slides per stool from three samples) were not feasible with the technical support available.

Having said that, the methods used were in accordance with the methods routinely used by the vector control division for assessing rates of infection across Uganda. To improve diagnostic accuracy, all slides were examined in the field for hookworm and reviewed the following week in the hospital laboratory for S. mansoni. Ten per cent of the slides were cross-checked at the Vector Control Division, Kampala, for quality control. Finally, individuals diagnosed with intestinal helminths were followed up and offered treatment, following current guidelines (WHO, 2006).

In addition, water-contact observations were carried out from approximately 6 am to $6 \mathrm{pm}$ on two consecutive days at ten of the fish landing sites. These observations documented the time of day of people's contact with river water, the body part immersed, length of immersion time and the activity being carried out. General observations were also 
made on each visit to the sites. Detailed water-contact observations were not carried out at two of the landing sites because the majority of users at these landing sites had moved elsewhere.

Throughout fieldwork, the findings were triangulated with additional sources where available. For example, laboratory records from local health centres, semi-structured interviews with key informants at the landing sites, interviews with district and health staff and ethnographic field notes of informal discussions and observations were all used to develop a broader picture of schistosomiasis infection levels, transmission and control.

Data collection and analysis was an iterative process. The focus of the survey was, in part, informed by previous ethnographic fieldwork in 2009; and the survey findings were explored through further ethnographic fieldwork. For example, responses to the questionnaire raised questions around people's access to, and experience of, the distribution of praziquantel; their use of Coartem for malaria; and the use of local remedies for various illnesses. These topics were subsequently explored through observations, and informal discussions, as well as semi-structured interviews. A descriptive analysis was carried out on the cross-sectional survey data and an inductive thematic analysis of field notes and interview transcripts was carried out manually.

Ethical approval for this research was granted through both the London School of Economics and Political Science and the National AIDS/HIV Research Committee (NARC) in Uganda for the Uganda National Council for Science and Technology (UNCST). Research clearance was granted by UNCST.

\section{Parasitological findings}

The overall prevalence of $S$. mansoni among the 383 people tested was $0.8 \%$. Three adults were found with $S$. mansoni at three sites: two women at two sites in Adjumani (aged 34 and 35 years) and one man at a site in Moyo (aged 22 years). One woman had a high-intensity infection (479 mean eggs per gram); the other two adults had a low-intensity infection (24 mean eggs per gram; 60 mean eggs per gram). At the individual fishing sites, the prevalence of $S$. mansoni among those tested was $2 \%, 3.5 \%$ and $3.5 \%$ respectively. At the other nine sites, the prevalence was $0 \%$. Additionally, hookworm was identified at three fishing sites. At two of these sites, one person (4\%) was diagnosed with hookworm at each site and, at the third site, four people $(11 \%)$ were diagnosed. Other soil-transmitted helminths were not found on microscopy.

While recent data on schistosomiasis diagnoses made outside a survey setting are limited, low levels of infection with $S$. mansoni were also seen at two local health centres near the river that had established laboratories. At one of these health centres, four cases had been detected out of approximately 500 people (men, women and children) who had had their stool examined in 2013. At the other health centre, the available laboratory registers from 2010 onwards showed that no $S$. mansoni had been detected on stool examination.

It is important to note that in Adjumani District, schistosomiasis was detected in inland areas away from the River Nile. At health centres, schistosomiasis was found on stool examination (evident from laboratory records and discussions with District Vector Control), and people were admitted to hospital with signs of chronic disease on 
ultrasound scans of the liver. Significantly, areas away from the River Nile in Adjumani District did not receive mass drug administration. Thus, while in Adjumani it appears that schistosomiasis is, in fact, still a problem in particular areas away from the River Nile, the available data suggest that the prevalence of $S$. mansoni has declined at sites along the river where the distribution of praziquantel has taken place.

On discussing the results of the survey with people at the fishing sites, a number doubted the findings as they still suffered from intestinal symptoms and had daily contact with the river water. In other words, the 'objective' rates of schistosomal infection as diagnosed on microscopy did not reflect people's experiences of suffering from intestinal illness. However, many other people corroborated the findings, recalling that schistosomiasis had been a significant health problem in the area before the distribution was introduced. In a group discussion, one fisherman described the symptoms people used to experience as 'stomach ache, dysentery and diarrhoea, and there were some people with swollen stomachs'. He went on to say: 'When people arrived from Sudan [in the late 1980s] it was common. When the distribution started, it started reducing.'

In sum, comparing levels of infection found at the beginning of mass drug administration in 2003 with ten years later in 2013, it appears that levels of infection with $S$. mansoni have declined. This raises the question: is mass drug administration successfully controlling intestinal schistosomiasis in this population?

\section{Drug uptake in Moyo and Adjumani Districts}

Mass drug administration has been the only public health intervention specifically implemented for controlling schistosomiasis in Moyo and Adjumani Districts. However, the distribution and self-reported consumption of praziquantel has been inconsistent. In Moyo District, praziquantel was reportedly distributed district-wide, first and foremost to primary schools and then to communities, if there was an adequate stock of praziquantel. In Adjumani District, it was only distributed to primary schools and villages located within two kilometres of the River Nile. Across both districts, praziquantel was distributed in villages for adults. At fishing sites that were not situated in a village people had to return to their home village to access the drugs. Furthermore, in both districts, community-wide distributions did not necessarily happen every year. Between April and August 2013, praziquantel was distributed in Moyo District; however, in Adjumani District, only ivermectin and albendazole were distributed for onchocerciasis and lymphatic filariasis. There are, therefore, differences between the fishing sites in the uptake of drugs from the distribution.

Findings from the questionnaire suggested that uptake of praziquantel was usually less than $75 \%$. Out of the 383 adults questioned, 214 reported having received praziquantel in 2013; that is, $56 \%$. This was $66 \%$ in Moyo District (139 of 210 participants), with rates varying between $41 \%$ and $79 \%$ across the sites. In Adjumani District, 43\% (75 of 173 participants) reported having received praziquantel in 2013, with rates varying from $34 \%$ to $58 \%$ across the sites. This finding is curious given that praziquantel was not distributed in the district in 2013. Ivermectin and albendazole had been distributed earlier in the year, and it is possible that some people's answers referred to this distribution. However, when subsequently questioned, people did differentiate between the two drug distributions. Praziquantel tablets are large, and have a pungent 
smell. Adults are typically given three or four tablets, depending on their height. They are distinct from ivermectin, which are small tablets, and albendazole, which is a large tablet but only one is given to each person. Other explanations as to how people in Adjumani District accessed praziquantel in 2013 were given. In some cases, people reported consuming tablets left over from previous years, and a few individuals in Adjumani District received praziquantel from the distribution in Moyo District.

Most people obtained praziquantel from the free distribution, distributed by voluntary community drug distributors, or members of the Village Health Team (VHT). One hundred and ninety-nine participants (95\%) in Moyo and 158 participants $(91 \%)$ in Adjumani reported having received praziquantel in this way at least once. Forty-nine participants (23\%) in Moyo, and $29(17 \%)$ in Adjumani, reported having taken praziquantel from private clinics or health centres at least once. Only a minority of people reported that they had never taken praziquantel: eight (4\%) in Moyo and six (4\%) in Adjumani. This was a concern for district staff. Commenting on this, one asked:

Why are certain people not interested in the free drug? Why are they not ready to take it?

Why do they not seek the drug when it is free and available from the VHT?

In further discussions with people at the river, it became clear that there were a number of reasons why this was the case. First, there were some people who remained reluctant to take praziquantel from the free distribution. In group discussions and conversations, some fisherfolk questioned the logic of the distribution when they did not feel unwell and had not been tested for the disease. To quote one fisherman: 'Why should I take the drug, if I am without disease?' Others questioned the need to take the drugs, stating that their parents and grandparents had lived healthily to an old age using the river water as they currently do. Secondly, it was reported that some people did not take praziquantel, as they had consumed alcohol at the time of the distribution. Thirdly, there were logistical constraints. Those who missed the distribution were often away at the time cultivating, fishing, taking produce to market or visiting relatives elsewhere. A group of fishermen described their problems in accessing the distribution:

Everybody knows about the taking of drugs, but if you are not found, for example because you are in the Nile, they say you go to [the distributor's] home. But you go maybe three times and they are not there so you miss. The drugs should be left at your place so that you can take them later. They don't distribute at [one site], they say to go to [the distributor's] home.

Accessing free medications thus presented a number of challenges to some people. Previous studies have also reported that people were reluctant to consume praziquantel due to concerns over the side-effects, particularly when praziquantel is taken without food (Parker \& Allen, 2011; Muhumuza et al., 2015). Although these concerns were registered in Moyo and Adjumani Districts, they did not necessarily deter people (Parker et al., 2012). Indeed, during the current research, the experience of side-effects demonstrated to some people the presence of intestinal worms and, therefore, the need to take praziquantel. To quote from one group discussion:

If you have [side-effects] it means you had the worms.

In many discussions about schistosomiasis and mass drug administration, a general perception was that the distribution had reduced the rate of disease, and that the drugs 
were needed, because people remained at risk along the river. Even though the exact transmission mechanism was not always known (with some saying that it was acquired by standing in the river or drinking the river water), it was known to be transmitted by, or along, the river. The majority of people thus took praziquantel from the community drug distributors, if it was available. While a few people were reluctant to take the drugs if they were not unwell, the vast majority of people were keen for the distribution to continue.

These findings resonate with previous research undertaken in the area, with Parker et al. (2012) and Allen and Storm (2012) suggesting that among the Madi of northwestern Uganda, there has been more generalized acceptance of the programme. A variety of historical, political and social issues contribute to this acceptance, including the legacy of colonial sleeping sickness control programmes, recent experiences of humanitarian relief programmes and the support of the Catholic Church.

In addition, while local healing practices and the use of local herbs (erua Madi) are an important part of health care choices in this area, they are often used alongside biomedical health care and they do not necessarily conflict with using biomedical health programmes (Allen \& Storm, 2012). For bilharzia (as schistosomiasis was commonly known), one fisherman explained, 'erua Madi a di dri uci uci' (translated as: 'local herbs are used, for what else is there to do?'). There were local herbs that are used to treat worms (obu) like schistosomiasis and, in the past, they were the main treatment. While people still use them today, this was rarely reported to be instead of mass drug administration. Rather, they were used when biomedical treatment was not available to them.

All these factors go some way to explaining people's perceptions of the mass drug administration programme. While there is demand for mass drug administration in this area, the questionnaire findings also demonstrate that actual uptake is variable within and between villages and over time. This raises the question: are low infection levels attributable to mass drug administration alone?

It is likely that mass treatment has had an impact. It is important to note, however, that in addition to the immediate effects of praziquantel treatment, there are other benefits that enhance the effectiveness of mass drug administration among adults. Dunne et al. (2006), for example, suggested that there are longer-term immunological effects providing some protection against future infection in adults. Age-related patterns of infection and responsiveness to praziquantel treatment (Kabatereine et al., 2004) may therefore have a role in explaining low levels of infection in this population. However, higher levels of infection and reinfection post-treatment have been seen among fishermen and women along Lake Albert and Lake Victoria despite mass drug administration (Kabatereine et al., 2011; Seto et al., 2012). This suggests that mass drug administration alone is not sufficient to control schistosomiasis.

Why does it appear to be different among fisherfolk in Moyo and Adjumani? What else has enabled low levels of infection in this area? The answers to these questions are found in the local context of schistosomiasis transmission and control.

\section{Additional explanations for the decline in the prevalence of $S$. mansoni}

There are three additional reasons that may help to explain why the prevalence of S. mansoni remains low. First, there may be significant anti-schistosomal properties of certain malaria treatments. It has been speculated that in co-endemic areas, artemisinin 
derivatives (artesunate and artemether) have additional benefits for schistosomiasis control alongside praziquantel treatment. This may be significant in Moyo and Adjumani Districts.

Although the number of studies on $S$. mansoni is small, it has been shown that repeated doses of artemisinin derivatives, in combination with praziquantel, have a prophylactic effect in reducing the risk of $S$. mansoni, and some benefit for cure rates (Utzinger et al., 2000; Pérez del Villar et al., 2012). Artemisinin has been incorporated into guidelines for preventing $S$. japonicum in China (Liu et al., 2011). However, due to concerns over the development of drug-resistant malaria, it is not recommended as a treatment or prevention strategy for schistosomiasis in co-endemic areas (ibid.). Outside interventions and trials, the influence on schistosomiasis control of artemisinin derivatives used for malaria in co-endemic areas is not known, although it has been suggested that there are additional benefits (Caffrey, 2007, p. 436; Keiser \& Utzinger, 2007; Pérez del Villar et al., 2012).

In Moyo and Adjumani Districts, artemisinin-based treatments were introduced as first-line malaria treatment in 2007. In the district hospitals, injectable artemisinin for severe malaria is not always available. The oral treatment Coartem (artemetherlumefantrine), the trade name that the artemisinin derivatives are commonly known by, is widely used. People source Coartem from government health centres or purchase it from private clinics. Some people use it when symptoms are interpreted as malaria, without seeking advice from a health centre or clinic, and whether a blood test for malaria is positive or negative. In villages near the river, even if Coartem is not available through the VHT, people are still able to access it from the health centres and private drug clinics.

From the questionnaire, 305 participants $(80 \%)$ reported that they had taken Coartem in 2013: 177 (84\%) in Moyo and 128 (74\%) in Adjumani. The majority of participants reported taking Coartem at least twice in the preceding year: that is, 256 participants $(67 \%)$ with an additional five participants $(1 \%)$ 'unsure' how many times they had taken it. In addition, six participants $(2 \%)$ reported that they were taking Coartem at the time of the survey, and $84(22 \%)$ said they had taken it in the preceding month. This high use of Coartem is in keeping with ethnographic observations of health care seeking and management, and with previous research on the use of biomedicine (Allen \& Storm, 2012).

Thus, while praziquantel use among participants in the cross-sectional survey was intermittent (normally once a year or less), self-reported Coartem use was higher. Overall, a larger proportion of participants had consumed Coartem in the preceding year compared with praziquantel. In addition, a large proportion of participants reported that they had taken Coartem multiple times in the year. If artemisinin-based compounds do have an additional impact on S. mansoni control in co-endemic areas, the high use of these drugs in addition to the consumption of praziquantel may have had a role in reducing the prevalence of schistosomiasis. However, Coartem is used in other areas where malaria and schistosomiasis are co-endemic, suggesting it may not be the only reason. In other words, additional factors need to be considered.

A second factor that might explain low levels of infection lies in the patterns of movement and migration in this border area. Previous anthropological research carried out in north-western Uganda indicated that movement across the Uganda/DRC border 
area may hinder schistosomiasis control (Parker et al., 2012). High rates of schistosomiasis were found at a market near Lake Albert and the border with the DRC. In contrast, low rates of schistosomiasis were found on a fishing island in Moyo District and it was suggested that these findings could in part be explained by differing movement patterns in these border areas and people's access to the free distribution of praziquantel. All those tested in Moyo District, despite being on the island, had accessed the distribution of drugs at some point, even if this had not occurred on an annual basis. The two sites differed, however, in other respects, one being a busy market setting, the other being a fishing island, and there were probably many local factors influencing the transmission of schistosomiasis at these sites. Despite the difference in settings, the contrasting findings are striking.

While these suggestions appear to still hold in Moyo and Adjumani Districts, it is a dynamic situation. For instance, over a few weeks in December 2013, tens of thousands of refugees from South Sudan settled in Adjumani District. This impacted on the local fish business, particularly at two of the landing sites nearest the settlements. These settlements grew quickly into bustling trading centres, and many women who had been engaged in the fish business at the landing sites sought other business at the settlements, such as preparing and selling cooked food. Subsequently, at one fishing site, a few secondary school girls arrived to begin fishmongering, seeking extra money for school fees, clothes and household needs. This all occurred just after completing the parasitology survey, and while it does not explain the parasitological findings, it highlights the challenges of schistosomiasis control in a region where political and economic issues result in constant movement and migration.

In addition, the everyday movement of people within the fish business has the potential to affect schistosomiasis transmission at the landing sites. The people involved in fishing-related activities waxes and wanes with seasonal changes in the fish catch and cultivation periods. One fisherman and local leader said:

You see, these people are like the Karamajong [in eastern Uganda]. The Karamajong, if there is nowhere to graze their cattle here, will move all the way up to Moyo and back. For these fishermen, some of them move all the way up that way or down that way. It depends where the fish are good. They may spend some days or weeks there, or come back daily.

This mobility was encountered during fieldwork. When returning to a landing site for follow-up, the majority of people who participated in the survey had moved to other sites, were taking fish to market or were in their fields cultivating crops. This has additional significance for understanding the parasitological findings. For instance, the woman identified with a high intensity of infection in the parasitological survey only used one fish landing site, but she spent part of the agricultural season in fields in the inland area of Adjumani District where schistosomiasis had been identified at the health centres. It is difficult to say where she contracted schistosomiasis; however, she did report taking praziquantel in May 2013 from a village drug distributor along the River Nile. This discussion on the movement of people between different areas highlights the importance of understanding the potential for transmission of schistosomiasis at the fishing sites.

Thus, a third consideration in explaining low levels of infection are the factors that affect transmission and lead to, or conversely, mitigate, risk of infection with $S$. mansoni at the fishing sites. Given the life cycle of $S$. mansoni, and the fact that transmission and exposure to infection are dependent on water contact, sanitation and hygiene practices, it 
is helpful to ask: what influences these behaviours? Are there behavioural or environmental reasons that can help explain the low prevalence of infection?

While access to safe water and sanitation reduces the probability of schistosomiasis infection (Grimes et al., 2014), associations between water, sanitation and hygiene on transmission are complex and depend on many contextual factors (Grimes et al., 2015). Even with improved access to safe water sources, preventing water contact completely is highly unlikely. Likewise, even with improved access to sanitation, transmission can continue unless water contact and open defecation are prevented completely (ibid.). These are important points to consider.

At many of the landing sites in Moyo and Adjumani, there is limited access to, or use of, sanitation facilities and safe water sources (borehole or piped water). At the time of the survey in 2013, the majority (eight) of the fishing sites had latrine blocks, yet at some sites the only option was to defecate in the bush surrounding the village. At two sites, previous attempts at constructing latrines had failed, with the structures quickly collapsing. Moreover, on the numerous small islands where there were no sanitation facilities, people reported having to defecate in the reeds or the river. In terms of safe water access, at the time of the survey, no landing sites had piped water, and at six landing sites there were no boreholes either - as such, people used the river water for all purposes. Even at landing sites with a nearby borehole, the river water continues to be used for certain activities. The cost of borehole water (which is paid as a monthly sum per household, typically 1000 Ugandan Shillings) is one limiting factor, as well as the time it takes women to fetch water. Women often collect river water for cleaning fish and washing clothes, not least because of the ease and its abundant supply. Many also prefer the river water because it is softer than the borehole water. Thus, despite access to borehole water, there are numerous reasons why people still use the river water.

However, exposure to infection with $S$. mansoni is dependent not simply on the availability or use of water sources per se, but on people's contact with river water. Transmission and infectivity are affected by the time of day of the water contact, the amount of time spent in the water and the degree of immersion in the water (Kloos et al., 1997). For instance, studies have demonstrated that peak concentrations of cercariae in the water occur around midday and in the early afternoon (Kazibwe et al., 2010). Although transmission can occur outside of this, there is a higher risk during this time period. The question that follows is: what influences patterns of water contact?

Typically, the fishermen go out to the river in the early morning, while women collect domestic water and the fishmongers arrive at the landing sites. In the mid- to latemorning hours, fishermen return from fishing and the fishmongers go to the boats to collect the fish. There is, therefore, a tendency for contact with the river to be during this period. At many sites, people have contact with the river water throughout the day, but at a number of the fish landing sites, this contact declines in the early afternoon. The female fishmongers remain at the landing sites cleaning and gutting fish and the fishermen return to the river to lay their nets in the late afternoon. It is likely that these patterns affect transmission - although it was also clear that there are large variations in people's water contact, making generalizations extremely difficult.

On the one hand, while people have contact with the river water at every fishing site, it is often avoided or brief. Fishermen take only a few steps in the water out to their canoes, while the women often make repeated but short trips into the river collecting 
water for cleaning fish or washing clothes. Furthermore, at sites where the fish catch is depleted, activities at the sites reduce and therefore people's contact with the river reduces. On the other hand, during the rainy season (which begins in late March or April and ends in late November), the water levels rise and the paths around the landing become water-logged. People have no choice but to wade, sometimes waist deep, out to their canoes. At many of the sites, female fishmongers tend to have more extensive and prolonged water contact. The bustle of collecting fish from the canoes, competition between fishmongers, and the weight of the basins of fish mean that women appear less cautious in avoiding water contact than the fishermen. At one site though, at the time of the water contact observations, it was the male fishmongers who spent sometimes an hour or more waist deep wading in the landing site while waiting for canoes to arrive early in the morning. And at another site, where the woman who had a high intensity of schistosomal infection came from, the women often spent ten to twenty minutes bathing in the landing site water in the hot afternoon hours.

Predominantly revolving around the fish business, the sites are sociable places where people pass time and women wash clothes while waiting for the fishermen to return to land. Therefore, at times, during women's contact with the river water, soap is used. This may have significance for the control of schistosomiasis as soap use during water contact is thought to provide some protection due to its toxic effect on the $S$. mansoni cercariae (reviewed in Grimes et al., 2015). It is thus possible that through this mechanism, risk of schistosomiasis is reduced, despite people's ongoing contact with the river water. Overall, certain aspects of water, sanitation and hygiene practices may mitigate the transmission of $S$. mansoni. Yet water contact and open defecation continue, so this too can only be a partial explanation.

Possibly, it is other factors that are more significant, none of which has been much investigated in the area, notably the local ecology of the snail intermediate hosts and their habitats. It is possible that the most common varieties of snails are not actually those that act as vectors. At the time of fieldwork, this had not been established, but some preliminary investigations by district health staff subsequently suggested that the intermediate host snail was found at a fishing site, although the infectivity of these hosts was not investigated. It is possible that something has occurred in the river here to make the location an unappealing habitat for the snails that are more conducive hosts and more prevalent upstream (Kazibwe et al., 2010). There have been seasonal and long-term changes in river flow and depth, which may have had ecological effects.

It is interesting to note that Bradley, reporting on Nelson's studies researching schistosomiasis in the area in the 1950s, described an absence of endemic cases in an area north of Rhino Camp (Bradley, 1975, p. 52). Although the exact location of these studies are not defined, research carried out more recently, and discussed earlier in this article, has found high prevalence rates in a similar area along the River Nile (Odongo-Aginyo et al., 2002, 2010). Possible explanations include ecological changes that affect transmission at certain points in time, and the micro-geographical patterns of infection and transmission, again influenced by ecological and social factors (De Moira et al., 2007).

The above discussion raises important points around the complexities of schistosomiasis transmission and control. By exploring these complexities, it is clear that an investigative approach that embraces these biological and social entanglements is required, a lesson that will be valuable elsewhere. 


\section{Conclusion: embracing complex realities}

The decline in the prevalence of $S$. mansoni along this stretch of the River Nile is both surprising and perplexing, raising more questions than answers. Consumption of praziquantel has surely contributed to the decline. Yet, comparing Moyo and Adjumani with other fishing areas in this region, where mass drug administration has occurred but rates of schistosomiasis remain high, suggests that we must look beyond the distribution of praziquantel. Possible explanations have been laid out in this article. It is likely that widespread and persistent use of artemisinin derivatives for treating malaria has also been important, and so have various ecological and social factors. There are other factors that add to the picture of schistosomiasis control, including the local ecology and presence of the snail host. It is impossible, based on the evidence available, to confirm any particular reason cited as causal or to quantify the magnitude of their effects. Nevertheless, this article highlights the need for more rigorous and substantial biosocial research than has hitherto been attempted. Up to now, surveys have neither asked, nor been able to answer, the questions that emerged through this inquiry. Yet given the complexities of schistosomiasis transmission and control, posing both biological and social questions is undoubtedly necessary. Recognizing this complexity, there is a need for similar biosocial research to occur in other parts of Uganda where prevalence remains high.

Looking forward, are low rates of infection sustainable over time? This question is pertinent, with the global health agenda shifting from reducing morbidity and controlling transmission, towards eliminating schistosomiasis as a matter of public health concern and ultimately ending transmission (WHO, 2013). There are undoubtedly limitations with relying on the distribution of free drugs for sustained schistosomiasis control. It is acknowledged that a combination of strategies is needed beyond mass drug administration, particularly in areas of low transmission (Rollinson et al., 2013; Grimes et al., 2015). In this regard, previous programmes have taken an integrated approach, most notably the Blue Nile Health Project in Sudan (for schistosomiasis and malaria) and a World Bank Loan Project in China (for S. japonicum). Still, we must be cautious when assessing the success of these programmes. Following the Blue Nile Health Project, levels of infection have increased again in some areas (Amin \& El Hussin, 2009; also discussed in Allen \& Parker, 2016). Likewise, in areas of China, similar concerns have been raised over the sustainability of control when control measures are relaxed (Xianyi et al., 2005). In Moyo and Adjumani, mass drug administration has been the only substantive public health intervention for schistosomiasis. As described in this article, just as there are factors that have enabled control, there are also factors that may tip the balance and enable resurgence in infection. Without doubt, biological and social questions, as explored in this article, need to be posed in order to understand the local complexities of sustainably controlling this disease. Finally, reflecting on the limited information on schistosomiasis transmission and control at the local level reveals broader issues around the kind of evidence informing policy. The discussion above emphasizes the importance of conducting biological and social research at the sites where mass drug administration has been introduced. This article has begun to map out some of the ways in which this may be done. 


\section{Acknowledgments}

The fieldwork was carried out as part of a $\mathrm{PhD}$ at the London School of Economics and Political Science (LSE), Department of International Development. It was supported financially by a Parkes Foundation PhD Grant and a LSE PhD scholarship. Local affiliation was with Professor Janet Seeley at the Social Science Research Programme MRC/UVRI Uganda Research Unit on AIDS. The author is grateful to Professors Tim Allen, Melissa Parker, Janet Seeley and Alison Elliott for their support and advice through various stages of the fieldwork, and Dr Edridah Muheki, Dr Narcis Kabatereine and David Oguttu at Vector Control Division, Kampala, who provided data, support and essential technical assistance for the parasitological survey. Adjumani and Moyo Hospitals kindly supported the work through use of microscopes and laboratory space and time. The author is also extremely grateful to the district staff and authorities in Adjumani and Moyo, including the District Health Officers. The District Vector Control Officers, Robert Dragule, Isaac Leku, Michael Nyaraga and Christopher Asiopkwe, helped carry out the parasitological survey alongside the laboratory technicians Pascale Zema, Peter Madra, Moses Otto and William Ambayo. Research assistants, Ronald Iya and Philip Goli carried out the questionnaires for the cross-sectional survey and were invaluable researchers throughout the fieldwork. Thanks also go to the driver for the survey team, Martin, and in particular to the Local Chairmen, BMU members and the men and women at the fish landing sites. Earlier versions of this article have been presented at conferences and seminars, with helpful comments and suggestions being received. The views expressed are solely those of the author.

\section{References}

Allen, T. (1991) The quest for therapy in 'Moyo District'. In Twaddle, M. \& Hansen, H. B. (eds) Changing Uganda: Dilemmas of Structural Adjustment and Revolutionary Change. James Currey, London, pp. 149-161.

Allen, T. \& Parker, M. (2011) The 'Other Diseases' of the Millennium Development Goals: rhetoric and reality of free drug distribution to cure the poor's parasites. Third World Quarterly 32(1), 91-117.

Allen, T. \& Parker, M. (2016) Deworming delusions? Mass drug administration in East African Schools. Journal of Biosocial Science 48(S1), S116-S147.

Allen, T. \& Storm, L. (2012) Quests for therapy in northern Uganda: healing at Laropi revisited. Journal of Eastern African Studies 6, 22-46.

Amin, M. A. \& El Hussin, D. M. (2009) Challenges in prevention and control of schistosomiasis in the Sudan. Sudan Journal of Medical Science 4(1), 79-83.

Beuving, J. (2010) Playing pool along the shores of Lake Victoria: fishermen, careers and capital accumulation in the Ugandan Nile perch business. Africa 80(2), 224-248.

Bradley, D. J. (1975) Schistosomiasis. In Hall, S. A. \& Langlands, B. W. (eds) Uganda Atlas of Disease Distribution. East African Publishing House Nairobi, pp. 50-53.

Caffrey, C. (2007) Chemotherapy of schistosomiasis: present and future. Current Opinion in Chemical Biology 11, 433-439.

De Moira, A. P., Fulford, A. J., Kabatereine, N. B., Kazibwe, F., Ouma, J. H., Dunne, D. W. \& Booth, M. (2007) Microgeographical and tribal variations in water contact and Schistosoma mansoni exposure within a Ugandan fishing community. Tropical Medicine \& International Health 12(6), 724-735. 
Dunne, D. W., Vennervald, B. J., Booth, M., Joseph, S., Fitzsimmons, C. M., Cahen, P. et al. (2006) Applied and basic research on the epidemiology, morbidity, and immunology of schistosomiasis in fishing communities on Lake Albert, Uganda. Transactions of the Royal Society of Tropical Medicine and Hygiene 100(3), 216-223.

Frenzel, K., Grigull, L., Odongo-Aginya, E., Ndugwa, C. M., Loroni-Lakwo, T., Schweigmann, U. et al. (1999) Evidence for a long-term effect of a single dose of praziquantel on Schistosoma mansoni-induced hepatosplenic lesions in northern Uganda. American Journal of Tropical Medicine and Hygiene 60(6), 927-931.

Grimes, J., Croll, D., Harrison, W., Utzinger, J., Freeman, M. \& Templeton, M. (2014) The relationship between water, sanitation and schistosomiasis: a systematic review and metaanalysis. PLoS Neglected Tropical Diseases 8(12), e3296.

Grimes, J., Croll, D., Harrison, W., Utzinger, J., Freeman, M. \& Templeton, M. (2015) The roles of water, sanitation and hygiene in reducing schistosomiasis: a review. Parasites and Vectors $\mathbf{8}(156)$.

Gryseels, B., Polman, K., Clerinx, J. \& Kestens, L. (2006) Human schistosomiasis. The Lancet 368, 1106-1118.

Kabatereine, N. B., Kemijumbi, J., Ouma, J. H., Kariuki, H. C., Richter, J., Kadzo, H. et al. (2004) Epidemiology and morbidity of Schistosoma mansoni infection in a fishing community along Lake Albert in Uganda. Transactions of the Royal Society of Tropical Medicine and Hygiene 98, 711-718.

Kabatereine, N. B., Standley, C. J., Sousa-Figueiredo, J. C., Fleming, F. M., Stothard, J. R., Talisuna, A. \& Fenwick, A. (2011) Integrated prevalence mapping of schistosomiasis, soiltransmitted helminthiasis and malaria in lakeside and island communities in Lake Victoria, Uganda. Parasites \& Vectors 4(4), 232.

Kabatereine, N. B., Tukahebwa, E., Kazibwe, F., Namwangye, H., Zaramba, S., Brooker, S. et al. (2006) Progress towards countrywide control of schistosomiasis and soil-transmitted helminthiasis in Uganda. Transactions of the Royal Society of Tropical Medicine and Hygiene 100(3) pp. 208-215.

Kazibwe, F., Makanga, B., Rubaire-Akiiki, C., Ouma, J., Kariuki, C., Kabatereine, N. B. et al. (2010) Transmission studies of intestinal schistosomiasis in Lake Albert, Uganda and experimental compatibility of local Biomphalaria spp. Parasitology International 59, 49-53.

Keiser, J. \& Utzinger, J. (2007) Artemisinins and synthetic trioxolanes in the treatment of helminth infections. Current Opinion in Infectious Diseases 20, 605-612.

King, C. (2010) Parasites and poverty: the case of schistosomiasis. Acta Tropica 113, 95-104.

Kloos, H., Fulford, A. J. C., Butterworth, A. E., Sturrock, R. F., Ouma, J. H., Kariuki, H. C. et al. (1997) Spatial patterns of human water contact and Schistosoma mansoni transmission and infection in four rural areas in Machakos District, Kenya. Social Science \& Medicine 44(7), 949-968.

Lamberton, P. H. L., Kabatereine, N. B., Oguttu, D. W., Fenwick, A. \& Webster, J. P. (2014) Sensitivity and specificity of multiple Kato-Katz thick smears and a circulating cathodic antigen test for Schistosoma mansoni diagnosis pre- and post-repeated-praziquantel treatment. PLoS Neglected Tropical Diseases 8, e3139.

Liu, R., Dong, H. F., Guo, Y., Zhao, Q-P. \& Jiang, M-S. (2011) Efficacy of praziquantel and artemisinin derivatives for the treatment and prevention of human schistosomiasis: a systematic review and meta-analysis. Parasites \& Vectors 4, 201.

Muhumuza, S., Olsen, A., Katahoire, A. \& Nuwaha, F. (2013) Uptake of preventive treatment for intestinal schistosomiasis among school children in Jinja District, Uganda: a cross sectional study. PLoS One 8(5), e63438.

Muhumuza, S., Olsen, A., Nuwaha, F. \& Katahoire, A. (2015) Understanding low uptake of mass treatment for intestinal schistosomiasis among school children: a qualitative study in Jinja District, Uganda. Journal of Biosocial Science 47(4), 505-520.

Odongo-Aginya, E. I., Grigull, L., Schweigmann, U., Loroni-Lakwo, T., Enrich, J. H., Gryseels, B. \& Doehring, E. (2002) High prevalence and morbidity of Schistosoma mansoni along the Albert Nile in Uganda. African Health Sciences 2(3), 99-106. 
Odongo-Aginya, E. I., Lakwo, T. L. \& Doehring, M. E. (2010) Evaluation of Schistosoma mansoni morbidity one year after praziquantel treatment in Rhino Camp and Obongi in West Nile, Uganda. African Journal of Infectious Diseases 4(2), 43-50.

Parker, M. \& Allen, T. (2011) Does mass drug administration for the integrated treatment of neglected tropical diseases really work? Assessing evidence for the control of schistosomiasis and soil-transmitted helminths in Uganda. Health Research Policy and Systems 9(3.

Parker, M. \& Allen, T. (2014) De-politicizing parasites: reflections on attempts to control the control of neglected tropical diseases. Medical Anthropology 33(3), 223-239.

Parker, M., Allen, T. \& Hastings, J. (2008) Resisting control of neglected tropical diseases: dilemmas in the mass treatment of schistosomiasis and soil-transmitted helminths in northwest Uganda. Journal of Biosocial Science 40, 161-181.

Parker, M., Allen, T., Pearson, G., Peach, N., Flynn, R. \& Rees, N. (2012) Border parasites: schistosomiasis control among Uganda's fisherfolk. Journal of Eastern African Studies 6, 98-123.

Pearson, G. (2009) Movement and marginality: understanding health as a collective responsibility and the mass treatment for neglected tropical disease in north-west Uganda. MSc Dissertation, Brunel University.

Pérez del Villar, L., Burguillo, F. J., López-Abán, J. \& Muro, A. (2012) Systematic review and meta-analysis of artemisinin based therapies for the treatment and prevention of schistosomiasis. PLoS One 7, e45867.

Rollinson, D., Knopp, S., Levitz, S., Stothard, J. R., Tchuem Tchuenté, L. A. et al. (2013) Time to set the agenda for schistosomiasis elimination. Acta Tropica 128, 423-440.

Seto, E. Y. W., Sousa-Figueiredo, J. C., Betson, M., Byalero, C., Kabatereine, N. B. \& Stothard, J. R. (2012) Patterns of intestinal schistosomiasis among mothers and young children from Lake Albert, Uganda: water contact and social networks inferred from wearable global positioning system dataloggers. Geospatial Health 7, 1-13.

UBOS (2014) National Population and Housing Census 2014. Provisional Results. Revised Edition. Uganda Bureau of Statistics.

Utzinger, J., N'Goran, E. K., N'Dri, A., Lengeler, C., Shuhua, X. \& Tanner, M. (2000) Oral artemether for prevention of Schistosoma mansoni infection: randomised controlled trial. The Lancet 355, 1320-1325.

Woodward, P. (1991) Uganda and southern Sudan 1986-9: new regimes and peripheral politics. In Hansen, H. \& Twaddle, M. (eds) Changing Uganda. James Currey, London, pp. 178-185.

WHO (1987) Uganda. Atlas of the Global Distribution of Schistosomiasis. WHO, Geneva, pp. 243-247.

WHO (2002) Prevention and control of schistosomiasis and soil-transmitted helminthiasis. WHO Technical Report Series 912, 1-57.

WHO (2006) Preventive Chemotherapy in Human Helminthiasis: Coordinated use of Antihelminthic Drugs in Control Interventions. A Manual for Health Professionals and Programme Managers. WHO, Geneva.

WHO (2012) Schistosomiasis: population requiring preventive chemotherapy and number of people treated in 2010. Weekly Epidemiological Record. No. 4 87, 37-44.

WHO (2013) Schistosomiasis Progress Report 2001-2011 and Strategic Plan 2012-2020. WHO, Geneva.

WHO (2016) Schistosomiasis: number of people treated worldwide in 2014. Weekly Epidemiological Record. No. 5 91, 53-60.

Xianyi, C., Wang, L., Cai, J., Zhou, X., Zheng, J., Guo, J. et al. (2005) Schistosomiasis control in China: the impact of a 10-year World Bank Loan Project (1992-2001). Bulletin of the World Health Organization 83(1), 43-48. 